\title{
Effects of Explosive Blast Trauma on Sleep Quality and Quality of Lives of the Workers in Ammunition Factory
}

\author{
Nuray Bayar Muluk, ${ }^{*, a}$ Ömer Oğuztürk, ${ }^{b}$ Osman Kürşat Arikan, ${ }^{c}$ Fulya Yalçınkaya, ${ }^{d}$ \\ Nursen Oral, ${ }^{e}$ and Ali Kemal Erdemoğlu ${ }^{f}$
}

${ }^{a}$ Faculty of Medicine, ENT Department, Kırlkkale University, Kırıkkale, Turkey, ${ }^{b}$ Faculty of Medicine, Psychiatry Department, Kırıkkale University, Kırlkkale, Turkey, ${ }^{c}$ Faculty of Medicine, ENT Department, Kırıkkale University, Kırıkkale, Turkey, ${ }^{d}$ Faculty of Medicine, Otolaryngology-Head and Neck Surgery Department, Division of Audiology and Speech Pathology, Hacettepe University, Ankara, Turkey ${ }^{e}$ Faculty of Medicine, Psychiatry Department, Gazi University, Ankara, Turkey and ${ }^{f}$ Faculty of Medicine, Neurology Department, Kırıkale University, Kırıkkale, Turkey

(Received February 8, 2009; Accepted May 30, 2009)

We investigated the effects of explosive acoustic trauma on sleep quality and quality of lives (QOLs) of the workers in Ammunition Factory. Forty-one male workers exposed to explosive acoustic trauma in Ammunition Factory 12 years ago constructed the study group (Group 1). Thirty-eight male workers of Ammunition Factory who were not exposed to the explosion, were included into the control group (Group 2). We used the Mini Sleep Questionnaire (MSQ) and SF-36 questionnaire. In workers of the study group who had experienced psychological and sleep problems; hearing loss and acute tinnitus just after explosion; and had higher education levels, some of the sleep and QOL parameters got worse. Workers, adjacent to the explosion site and not used earpluggs during explosion, had more impaired sleep quality results $(p<0.05)$. We may conclude that acute explosive acoustic trauma may affect negatively some items of sleep quality and quality of the lives of the workers. Workers should be educated to use ear muffs and ear plugs. For post-explosion sleep problems and other psychological problems, psychological support should be given to the workers by the health-team and their families.

Key words — explosive acoustic trauma, sleep quality, mini sleep questionnaire (MSQ), SF-36 health survey, quality of life (QOL)

\section{INTRODUCTION}

Acute acoustic trauma (AAT) is relatively common after exposure to intense gunfire noise which consist of a combination of energies from acoustic and pressure waves at high intensities, resulting in rupture of the organ of Corti, its separation from the basilar membrane and fracture and displacement of hair cell sterocilia. ${ }^{1,2)}$ Based on impaired cochlear blood flow and cochlear hypoxia may be the causing mechanisms for AAT. ${ }^{3)}$

AATs are caused by exposure to extremely high noise levels ranging from milliseconds to several hours' duration. Their pathogenesis is assumed to consist of micromechanical-traumatic and biochemical-metabolic damage to the outer hair cells. ${ }^{1)}$ The specific effects, caused by the noise ex-

${ }^{*}$ To whom correspondence should be addressed: Birlik Mahallesi, Zirvekent 2. Etap Sitesi, C-3 blok, No: 62/43 06610 Çankaya, Ankara, Turkey. Tel.: +90-312-4964073; Fax: +90-

318-2252819; E-mail: nbayarmuluk@yahoo.com posure, affect the auditory system (acoustic trauma, auditory weariness, noise deafness) and the vestibular system (vertigo, sickness, trouble of balance). ${ }^{4}$ )

After AAT, the main threshold shift started at $1 \mathrm{kHz}$ in $8 \%$, at $2 \mathrm{kHz}$ in $49 \%$, at $4 \mathrm{kHz}$ in $19 \%$ and at $6 \mathrm{kHz}$ in $6 \%$ of the ears. ${ }^{5}$ It was reported that the AATs occurred in unprotected ears, resulting in symptoms such as tinnitus and/or hearing impairment. $46.7 \%$ of conscripts had hearing impairment and $94.2 \%$ tinnitus. Hearing loss, tinnitus or both were experienced by $45 \%$ of conscripts at the last follow-up. $\left.{ }^{6}\right)$ Oeken ${ }^{7)}$ investigated the changes to the distortion products of otoacoustic emissions (DPOAE) in seventeen patients were examined after sustaining AAT. DPOAE seemed to indicate the likelihood of recovery of hearing threshold after an AAT.

Noise is considered to be a non-specific stressor which generally causes physiological and psychological effects in an individual. Many occupations involve workers being subjected to loud noise lev- 
els without adequate protective measures. It was reported that workers exposed to loud background occupational noise are at an increased risk of having poor quality sleep but adaptation to this effect probably takes place after a few years. ${ }^{8)}$ Noise produces discomfort, diminishes attention, or impedes communication, concentration and sleep. ${ }^{9)}$

In Kırıkkale, at Ammunition Factory, founded in 1925, the first important explosion has occurred in June, 18th, 1986. In military munition depot near Yahşihan, town of Kırıkkale, the boom was happened because of extreme temperatures, nobody was lost; and 19 people were injured. The second explosion has occurred two months later and 7 firefighters had died. Fire in Trinitrotoluene (TNT) Atelier, in which 350 workers employed, caused strong explosions in building containing 2.5 tons of TNT. In February 2nd 1988, explosion was occurred in Ammunition factory. There was not any loss. In filling atelier, financial damage occurred. In July 3rd 1997, 2 people lost their lives in the explosion of the Ammunition factory. There were 130 workers who were injured due to explosion. Total of one thousand and seven hundred and fifty workers were present in the factory during the explosion was occurred. On the day of the explosion, there was no authorized employee of the Ammunition Factory. Four hundred and fifty people were exposed to the explosion. The other workers were in the different parts of the factory and they were not exposed to the explosion. Explosion was occured during the lunch-time. In the Bomb-filling Atelier, while TNT was filling into the bomb, explosion was happened. To intervene immediately after the explosion, fire crew extinguished the fire with difficulties. Just in case, all city center and the places around the city was emptied by governorship. By the impact of explosion, surrounding houses' walls were collapsed, roof of barns were collapsed in farms, and bovine animals were injured.

In the present study, we investigated the effects of explosive blast trauma on sleep qualities and quality of lives (QOLs) of the workers exposed blast trauma during their work in Ammunition Factory in 1997. We investigated the sleep qualities and QOLs of the workers by Mini Sleep Questionnaire $(\mathrm{MSQ})^{10)}$ and Short Form (SF)-36 Health Survey ${ }^{11)}$ respectively. In the literature, there are not any studies about this matter.

\section{MATERIALS AND METHODS}

This prospective study was carried out in the Ear Nose Throat (ENT) and Psychiatry Departments of Kırıkkale University Faculty of Medicine in 2007. Subjects — The study was carried out in patients exposed to explosive blast trauma during their works in Ammunition Factory in 1997. Total of one thousand and seven hundred and fifty workers were present in the factory during the explosion was occurred. On the day of the explosion, there was no authorized employee of the Ammunition Factory. Four hundred and fifty people were exposed to the explosion. The other workers were in the different parts of the factory and they were not exposed to the explosion. Explosion was occured during the lunch-time. In the Bomb-filling Atelier, while TNT was filling into the bomb, explosion was happened.

To intervene immediately after the explosion, fire crew extinguished the fire with difficulties. Just in case, all city center and the places around the city was emptied by governorship. In the explosion, number of fatalities was 2 . There were 130 workers who were injured due to explosion.

The study group was consisted of the workers exposed to explosion and accepted to participate in the study. Forty-one male workers exposed to explosive acoustic trauma in Ammunition Factory 12 years ago constructed the study group (Group 1). They were included into the study with their agreement by written informed consent to participate the study, and to give permission for the use of their all data. Their mean age was $45.17 \pm 5.61$ (Ranged from 34 to 64). The workers were evaluated by periodic health check-up in the factory. In the factory, the noise level in the factory was measured and the noise level map of the all departments in the factory was made. Noise levels varied between $70 \mathrm{~dB}$ and $100 \mathrm{~dB}$. There were no ototoxic chemical exposures in the factory. The workers were instructed to wear hearing protection devices (protective ear muffs or earplugs).

In Turkey, according to Occupational Law, the highest noise levels acceptable in terms of hearing health are identified by noise control regulations (See on Table 1). According to the Noise Regulations; when noise exposure values exceed the acceptable lowest exposure of $80 \mathrm{~dB}$, the employer will provide preventive measures in ready to use, such as earplugs and earmuffs.

The control group was consisted of the workers, not exposed to explosion, and agreed to participate 
Table 1. The Highest Noise Levels Acceptable in Terms of Hearing Health in Turkey

\begin{tabular}{cc}
\hline \hline Maximum Noise Level $(\mathrm{dB})$ & $\begin{array}{c}\text { Noise exposure time } \\
(\mathrm{hr} / \text { day })\end{array}$ \\
\hline 80 & 7.5 \\
90 & 4 \\
95 & 2 \\
100 & 1 \\
105 & $0.5(30 \mathrm{~min} / \mathrm{day})$ \\
110 & $0.25(15 \mathrm{~min} /$ day $)$ \\
115 & $1 / 8(7.5 \mathrm{~min} /$ day $)$ \\
\hline
\end{tabular}

in the study. Thirty-eight male workers of Ammunition Factory, who were not exposed to the explosion, were included into the control group (Group 2). Control groups ages were matched with the study group. Their mean age was $44.18 \pm 6.38$ (Ranged from 28 to 53).

All workers' job contents in the study and control groups and working schedule were both same at the Ammunition Factory.

The criteria for exclusion from the study: Any of the patients in the study and control group had head trauma; and in the present time, any symptoms and findings of the infectious ear diseases. None of them had obstructive sleep apnea and known psychiatric diseases.

Instrumentation - Questionnaire: Education, daily noise exposure (hour), total noise exposure time (years), usage of ear muffs, usage of ear plugs, presence of earplugs during explosion, psychological problems after explosion, sleep disorders after explosion, acute tinnitus during and just after explosion, presence of chronic tinnitus still going on, hearing loss after explosion, distance from the explosion site (meters) and Brinkman Index ${ }^{12)}$ values were evaluated.

$M S Q$ : A 10-point MSQ was completed focusing specifically on the sleep complaint. This consisted of questions about fatigue, daytime sleep, restless sleep, sleep medications, and other factors disturbing or affecting sleep. Items about sleep quality were indicated on a frequency scale of $1-7$ ( $1=$ never, 4 = sometimes, 7 = always $)$. Mean scores in normal sleepers across different age groups was 2.1-2.5 with Standard deviation of 1.3 or 1.4. Items of 10-point MSQ are:1-Sleep delay (SD), 2-Sleep awakenings (SA), 3-Sleep medications (SM), 4-Daytime sleep (DS), 5-Morning fatigue (MF), 6-Habitual snoring (HS), 7-Morningawakening (MA), 8-Morning headache ( $\left.\mathrm{MH}^{\mathrm{MSQ}}\right)$,
9-Chronic fatigue (CF) and 10-Restless Sleep (RS). ${ }^{10)}$

The SF-36 Health Survey: The SF-36 Health Survey ${ }^{11)}$ is a multi-item global assessment of patient function that assesses eight health concepts including: Physical functioning (10 items) (PF); Role limitations due to physical problems (four items) (RP); Social functioning (two items) (SF); Bodily pain (two items) (BP); General mental health (five items) $\left(\mathrm{MH}^{\mathrm{SF}-36}\right)$; Role limitations de to emotional problems (three items) (RE); Vitality (four items) (VT); and General health perceptions (six items) $(\mathrm{GH})$.

Each scale yields a score of 0-100, with lower scores reflecting greater limitations in function.

All steps of the study were planned and continued according to the principles outlined in the Declaration of Helsinki. ${ }^{13)}$ Permission for the study was taken from Rectorship of Kırıkkale University and Directorate General of Ammunition Factory.

Statistical Analysis — Statistical packet for SPSS (Version 8.0) was used for statistical evaluation. The difference between age; and each of the MSQ results (SD, SA, SM, DS, MF, HS, MA, $\mathrm{MH}^{\mathrm{MSQ}}, \mathrm{CF}, \mathrm{RS}$ ); and each of the SF-36 Health Survey results (PF, RP, SF, BP, MH ${ }^{\mathrm{SF}-36}$, RE, VT, $\mathrm{GH}$ ) of study (Exposed to explosion group workersGroup 1) and control groups (Non-exposed to explosion group workers- Group 2) were analyzed by "Student- $t$ Test."

For the study group, correlation for each of the education, usage of ear muffs, usage of ear plugs, presence of earplugs during explosion, psychological problems after explosion, sleep disorders after explosion, acute tinnitus during and after explosion, presence of tinnitus still going on, hearing loss after explosion on each of the SF-36 Health Survey Items and MSQ Items were analyzed by "Spearman's Correlation Rho Efficient."

For the study group, correlation for each of the daily noise exposure (hour), total noise exposure (years), distance from the explosion site and Brinkman Index on each of the SF-36 Health Survey Items and MSQ Items were analyzed by "Pearson Correlation". $p$ value $<0.05$ was considered statistically significant.

\section{RESULTS}

Education level of the study group was primary school (7.3\%), secondary school (78\%), high school 
$(4.8 \%)$ and university $(4.8 \%)$. Ear muffs usage of the workers were never $(80.5 \%)$; rarely $(17.0 \%)$; and often $(2.5 \%)$. Ear plugs usage of the workers were never (63.5\%); rarely (29.2\%); often $(4.8 \%)$; and always $(2.5 \%)$. Only one worker $(2.4 \%)$ had been using earplugs during explosion. Acute tinnitus during and just after explosion was present in $51 \%$ of the workers and hearing loss after explosion was present $19.5 \%$ of the workers. Chronic tinnitus still going on was present $34.1 \%$ of the workers. Psychological problems (14.6\%) and sleep disorders after explosion (14.6\%) were also present.

Age, MSQ and SF-36 Health Survey Results of the study and control groups are demonstrated on Table 2.

The difference between age; and each of the MSQ results (SD, SA, SM, DS, MF, HS, MA, $\mathrm{MH}^{\mathrm{MSQ}}, \mathrm{CF}, \mathrm{RS}$ ); and each of the SF-36 Health Survey results (PF, RP, SF, BP, MH $\left.{ }^{\mathrm{SF}-36}, \mathrm{RE}, \mathrm{VT}, \mathrm{GH}\right)$ of study (Group 1) and control groups (Group 2) were analyzed by "Student-t Test". The difference between all items of 10-point MSQ and SF-36 items were not significant between two groups $(p>0.05)$.

For the study group, the results of Pearson Correlation and Spearman's Correlation Rho Efficient Tests for SF-36 items (Table 3) showed that:
Education level being increased, RE values significantly decreased $(p=0.043)$. With psychological problems after explosion, $\mathrm{PF}(p=0.011)$, $\mathrm{SF}(p=0.008), \mathrm{GH}(p=0.009)$ significantly decreased. Other SF-36 items also non-significantly decreased $(p>0.05)$. With sleep disorders after explosion, $\mathrm{PF}(p=0.001)$ and $\mathrm{GH}(p=0.001)$ significantly decreased. Acute tinnitus during and just after explosion being present, there were significant decrease in $\operatorname{SF}(p=0.002)$. In workers with chronic tinnitus is still going on, no significant decrease was detected in SF-36 items $(p>0.05)$. Hearing loss after explosion being occurred, GH value significantly decreased $(p=0.020)$. Distance from the explosion site getting nearer; except BF and VT, other SF-36 items insignificantly decreased $(p>0.05)$.

For the study group, the results of Pearson Correlation and Spearman's Correlation Rho Efficient Test for MSQ Items (Table 4) showed that:

Education level being increased, SM values significantly increased $(p=0.001)$. Daily noise exposure being increased, all MSQ items nonsignificantly increased $(p>0.05)$. Total noise exposure time being increased, SD $(p=0.030)$, $\mathrm{SM}(p=0.007)$, HS $(p=0.040)$ and $\mathrm{MH}(p=$ $0.021)$ increased; therefore sleep quality decreased.

Table 2. Age, MSQ and SF-36 Health Survey Results of the Group 1 and 2

\begin{tabular}{|c|c|c|c|c|c|c|c|c|}
\hline & \multicolumn{8}{|c|}{ Groups } \\
\hline & \multicolumn{4}{|c|}{ Group $1(n=41)$} & \multicolumn{4}{|c|}{ Group $2(n=38)$} \\
\hline & Mean & St.Dev. & Minimum & Maksimum & Mean & St.Dev. & Minimum & Maksimum \\
\hline Age & 45.17 & 5.61 & 34.00 & 64.00 & 44.18 & 6.38 & 28.00 & 53.00 \\
\hline \multicolumn{9}{|c|}{ MSQ Items } \\
\hline SD & 3.56 & 1.71 & 1.00 & 7.00 & 3.21 & 1.66 & 1.00 & 7.00 \\
\hline SA & 3.78 & 1.69 & 1.00 & 7.00 & 3.60 & 1.73 & 1.00 & 7.00 \\
\hline SM & 1.12 & 0.55 & 1.00 & 4.00 & 1.00 & 0.00 & 1.00 & 1.00 \\
\hline DS & 3.92 & 1.25 & 1.00 & 7.00 & 3.36 & 1.23 & 1.00 & 4.00 \\
\hline MF & 3.92 & 1.25 & 1.00 & 7.00 & 3.44 & 1.36 & 1.00 & 7.00 \\
\hline HS & 4.43 & 1.84 & 1.00 & 7.00 & 4.00 & 1.97 & 1.00 & 7.00 \\
\hline MA & 3.70 & 1.87 & 1.00 & 7.00 & 3.52 & 1.78 & 1.00 & 7.00 \\
\hline $\mathrm{MH}$ & 2.39 & 1.51 & 1.00 & 4.00 & 2.57 & 1.94 & 1.00 & 7.00 \\
\hline $\mathrm{CF}$ & 4.00 & 1.64 & 1.00 & 7.00 & 3.52 & 1.78 & 1.00 & 7.00 \\
\hline RS & 2.90 & 1.46 & 1.00 & 4.00 & 2.57 & 1.81 & 1.00 & 7.00 \\
\hline \multicolumn{9}{|c|}{ Results of the SF-36 } \\
\hline$\overline{P F}$ & 86.34 & 15.37 & 30.00 & 100.00 & 83.42 & 20.20 & 20.00 & 100.00 \\
\hline $\mathrm{RP}$ & 67.07 & 41.62 & 0.00 & 100.00 & 82.89 & 30.81 & 0.00 & 100.00 \\
\hline SF & 81.70 & 21.48 & 25.00 & 100.00 & 84.21 & 17.84 & 37.50 & 100.00 \\
\hline $\mathrm{BP}$ & 74.62 & 24.69 & 10.00 & 100.00 & 80.06 & 18.49 & 35.00 & 100.00 \\
\hline $\mathrm{MH}$ & 74.87 & 13.20 & 32.00 & 100.00 & 69.43 & 23.27 & 12.00 & 100.00 \\
\hline $\mathrm{RE}$ & 85.36 & 32.53 & 0.00 & 100.00 & 95.61 & 11.42 & 66.66 & 100.00 \\
\hline VT & 66.70 & 19.98 & 25.00 & 100.00 & 69.73 & 18.30 & 25.00 & 100.00 \\
\hline $\mathrm{GH}$ & 68.75 & 19.20 & 12.50 & 95.83 & 66.20 & 18.04 & 16.66 & 91.66 \\
\hline
\end{tabular}


Table 3. In the Study Group (Exposed to explosive blast trauma), Pearson Correlation Test or Spearman's Correlation Rho Efficient Test Results of the Factors on SF-36 Survey Results

\begin{tabular}{|c|c|c|c|c|c|c|c|c|}
\hline & \multicolumn{8}{|c|}{ SF-36 Survey Results } \\
\hline & \multicolumn{2}{|c|}{$\mathrm{PF}$} & \multicolumn{2}{|c|}{ RP } & \multicolumn{2}{|c|}{$\overline{\mathrm{SF}}$} & \multicolumn{2}{|c|}{$\mathrm{BP}$} \\
\hline & $r$ & $p$ & $r$ & $p$ & $r$ & $p$ & $r$ & $p$ \\
\hline Education $^{a)}$ & 0.082 & 0.622 & -0.077 & 0.639 & 0.044 & 0.789 & -0.265 & 0.103 \\
\hline Daily noise exposure $(h)^{b)}$ & -0.174 & 0.284 & -0.191 & 0.237 & 0.004 & 0.983 & 0.117 & 0.473 \\
\hline Total noise exposure (Years) ${ }^{b}$ & -0.178 & 0.272 & 0.114 & 0.483 & -0.065 & 0.691 & 0.003 & 0.984 \\
\hline Usage of ear muffs ${ }^{a)}$ & -0.095 & 0.556 & -0.054 & 0.736 & 0.030 & 0.853 & 0.149 & 0.353 \\
\hline Usage of ear plugs ${ }^{a)}$ & -0.010 & 0.949 & 0.117 & 0.467 & -0.031 & 0.850 & -0.112 & 0.487 \\
\hline $\begin{array}{l}\text { Absence of earplugs during } \\
\text { explosion }^{a)}\end{array}$ & -0.171 & 0.284 & 0.133 & 0.406 & -0.085 & 0.598 & -0.020 & 0.900 \\
\hline $\begin{array}{l}\text { Psychological problems after } \\
\text { explosion }^{a)}\end{array}$ & -0.395 & 0.011 & -0.268 & 0.091 & -0.410 & 0.008 & -0.221 & 0.164 \\
\hline Sleep disorders after exeplosion ${ }^{a}$ & -0.499 & 0.001 & -0.278 & 0.078 & -0.292 & 0.064 & -0.263 & 0.096 \\
\hline $\begin{array}{l}\text { Acute tinnitus during and just } \\
\text { after explosion }{ }^{a}\end{array}$ & -0.091 & 0.572 & -0.119 & 0.459 & -0.461 & 0.002 & -0.023 & 0.887 \\
\hline Chronic tinnitus still going on ${ }^{a)}$ & -0.214 & 0.179 & -0.043 & 0.788 & 0.131 & 0.414 & 0.167 & 0.296 \\
\hline Hearing loss after explosion ${ }^{a)}$ & -0.341 & 0.029 & -0.254 & 0.109 & -0.234 & 0.141 & -0.145 & 0.366 \\
\hline Distance from the explosion site ${ }^{b)}$ & 0.074 & 0.647 & 0.086 & 0.594 & 0.036 & 0.824 & -0.096 & 0.551 \\
\hline \multirow[t]{4}{*}{ Brinkmann Index $^{b)}$} & -0.096 & 0.552 & -0.188 & 0.238 & -0.262 & 0.098 & 0.181 & 0.256 \\
\hline & \multicolumn{8}{|c|}{ SF-36 Survey Results } \\
\hline & \multicolumn{2}{|c|}{$\mathrm{MH}^{\mathrm{SF}-36}$} & \multicolumn{2}{|c|}{ RE } & \multicolumn{2}{|c|}{$\mathrm{VT}$} & \multicolumn{2}{|c|}{$\mathrm{GH}$} \\
\hline & $r$ & $p$ & $r$ & $p$ & $r$ & $p$ & $r$ & $p$ \\
\hline Education $^{a}$ & 0.055 & 0.741 & -0.326 & 0.043 & 0.185 & 0.259 & 0.008 & 0.961 \\
\hline Daily noise exposure $(\mathrm{h})^{b)}$ & 0.008 & 0.960 & 0.098 & 0.546 & -0.093 & 0.568 & -0.028 & 0.863 \\
\hline Total noise exposure (Years) ${ }^{b}$ & 0.172 & 0.289 & 0.095 & 0.559 & 0.186 & 0.250 & 0.071 & 0.662 \\
\hline Usage of ear muffs ${ }^{a)}$ & -0.032 & 0.843 & 0.065 & 0.688 & -0.071 & 0.661 & 0.110 & 0.495 \\
\hline Usage of ear plugs ${ }^{a)}$ & -0.063 & 0.695 & 0.075 & 0.639 & -0.018 & 0.909 & 0.022 & 0.892 \\
\hline $\begin{array}{l}\text { Absence of earplugs during } \\
\text { explosion }^{a}\end{array}$ & 0.134 & 0.402 & 0.077 & 0.631 & 0.235 & 0.138 & -0.047 & 0.770 \\
\hline $\begin{array}{l}\text { Psychological problems after } \\
\text { explosion }^{a)}\end{array}$ & -0.231 & 0.146 & -0.226 & 0.156 & -0.226 & 0.156 & -0.404 & 0.009 \\
\hline Sleep disorders after exeplosion ${ }^{a}$ & -0.298 & 0.059 & 0.201 & 0.209 & -0.255 & 0.108 & -0.481 & 0.001 \\
\hline $\begin{array}{l}\text { Acute tinnitus during and just } \\
\text { after explosion }{ }^{a)}\end{array}$ & -0.305 & 0.053 & -0.122 & 0.446 & -0.087 & 0.588 & -0.199 & 0.212 \\
\hline Chronic tinnitus still going on ${ }^{a)}$ & 0.238 & 0.134 & 0.211 & 0.186 & 0.092 & 0.568 & 0.066 & 0.684 \\
\hline Hearing loss after explosion ${ }^{a)}$ & -0.170 & 0.288 & -0.075 & 0.640 & -0.170 & 0.287 & -0.361 & 0.020 \\
\hline Distance from the explosion site ${ }^{b)}$ & 0.150 & 0.349 & 0.096 & 0.549 & -0.009 & 0.954 & 0.229 & 0.149 \\
\hline Brinkmann Index ${ }^{b)}$ & -0.071 & 0.659 & -0.099 & 0.536 & -0.124 & 0.439 & -0.220 & 0.166 \\
\hline
\end{tabular}

a) Spearman's Correlation Rho Efficient Test was used for statistical analysis. b) Pearson Correlation Test was used for statistical analysis.

Earplugs being not present during explosion, SM $(p=0.000)$ and MF $(p=0.012)$ significantly increased. With psychological problems after explosion, $\mathrm{MF}(p=0.039)$, HS $(p=0.027)$ and $\mathrm{RS}$ $(p=0.017)$ significantly increased. With sleep disorders after explosion, SD $(p=0.004)$, SA $(p=0.009)$, MF $(p=0.030)$, HS $(p=0.034)$ and RS $(p=0.004)$ significantly increased. Acute tinnitus during and just after explosion being present, there were significant increase in SD $(p=0.003)$, SA $(p=0.049)$, HS $(p=0.046)$ and MA $(p=$
$0.037)$ items of MSQ. In workers with chronic tinnitus is still going on, HS ( $p=0.027)$ significantly increased. Hearing loss after explosion being occurred, SD value significantly increased ( $p=$ $0.029)$. Other MSQ items insignificantly increased $(p>0.05)$. Distance from the explosion site getting nearer, SA value significantly increased ( $p=$ $0.023)$. SD, SM, DS, MF, HS and MH values insignificantly increased $(p>0.05)$. Brinkman Index being increased, HS value significantly increased $(p=0.030)$. 
Table 4. In the Study Group (Exposed to explosive blast trauma), Pearson Correlation Test or Spearman's Correlation Rho Efficient Test Results of the Factors on MSQ Items

\begin{tabular}{|c|c|c|c|c|c|c|c|c|c|c|}
\hline & \multicolumn{10}{|c|}{ MSQ Items } \\
\hline & \multicolumn{2}{|c|}{$\mathrm{SD}$} & \multicolumn{2}{|c|}{ SA } & \multicolumn{2}{|c|}{ SM } & \multicolumn{2}{|c|}{ DS } & \multicolumn{2}{|r|}{$\mathrm{MF}$} \\
\hline & $r$ & $p$ & $r$ & $p$ & $r$ & $p$ & $r$ & $p$ & $r$ & $p$ \\
\hline Education $^{a)}$ & 0.269 & 0.098 & 0.206 & 0.209 & 0.513 & 0.001 & 0.124 & 0.452 & -0.132 & 0.425 \\
\hline Daily noise exposure $(\mathrm{hr})^{b)}$ & 0.145 & 0.373 & 0.174 & 0.284 & 0.004 & 0.978 & 0.162 & 0.319 & 0.334 & 0.035 \\
\hline Total noise exposure (years) $)^{b)}$ & 0.343 & 0.030 & 0.228 & 0.157 & 0.423 & 0.007 & -0.251 & 0.119 & 0.296 & 0.064 \\
\hline Usage of ear muffs ${ }^{a)}$ & 0.193 & 0.226 & 0.101 & 0.529 & -0.169 & 0.291 & -0.178 & 0.265 & 0.155 & 0.332 \\
\hline Usage of ear plugs ${ }^{a)}$ & 0.243 & 0.126 & 0.057 & 0.722 & 0.160 & 0.318 & -0.145 & 0.367 & 0.318 & 0.043 \\
\hline $\begin{array}{l}\text { Absence of earplugs during } \\
\text { explosion }^{a)}\end{array}$ & 0.296 & 0.061 & 0.293 & 0.063 & 0.680 & 0.000 & 0.010 & 0.950 & 0.388 & 0.012 \\
\hline $\begin{array}{l}\text { Psychological problems after } \\
\text { explosion }^{a}\end{array}$ & 0.302 & 0.055 & 0.265 & 0.094 & 0.126 & 0.432 & -0.106 & 0.511 & 0.323 & 0.039 \\
\hline Sleep disorders after explosion ${ }^{a}$ & 0.441 & 0.004 & 0.406 & 0.009 & 0.106 & 0.510 & 0.141 & 0.378 & 0.340 & 0.030 \\
\hline $\begin{array}{l}\text { Acute tinnitus during and just } \\
\text { after explosion }^{a} \text { ) }\end{array}$ & 0.449 & 0.003 & 0.309 & 0.049 & 0.221 & 0.165 & -0.057 & 0.725 & 0.296 & 0.060 \\
\hline Chronic tinnitus still going on ${ }^{a)}$ & 0.187 & 0.241 & 0.183 & 0.252 & 0.070 & 0.664 & -0.083 & 0.606 & 0.040 & 0.805 \\
\hline Hearing loss after explosion ${ }^{a}$ & 0.342 & 0.029 & 0.171 & 0.284 & 0.167 & 0.296 & 0.028 & 0.863 & 0.179 & 0.263 \\
\hline Distance from the explosion site ${ }^{b)}$ & -0.050 & 0.743 & -0.365 & 0.023 & -0.104 & 0.516 & -0.050 & 0.754 & -0.085 & 0.597 \\
\hline \multirow[t]{4}{*}{ Brinkmann Index ${ }^{b)}$} & 0.005 & 0.977 & 0.005 & 0.556 & -0.039 & 0.809 & 0.202 & 0.206 & -0.057 & 0.723 \\
\hline & \multicolumn{10}{|c|}{ MSQ Items } \\
\hline & \multicolumn{2}{|c|}{ HS } & \multicolumn{2}{|c|}{ MA } & \multicolumn{2}{|c|}{ MH } & \multicolumn{2}{|c|}{$\mathrm{CF}$} & \multicolumn{2}{|c|}{$\mathrm{RS}$} \\
\hline & $r$ & $p$ & $r$ & $p$ & $r$ & $p$ & $r$ & $p$ & $r$ & $p$ \\
\hline Education $^{a)}$ & -0.197 & 0.229 & -0.088 & 0.595 & -0.114 & 0.490 & 0.114 & 0.489 & 0.121 & 0.462 \\
\hline Daily noise exposure $(\mathrm{hr})^{b)}$ & 0.251 & 0.118 & 0.081 & 0.621 & 0.250 & 0.120 & 0.189 & 0.243 & 0.148 & 0.363 \\
\hline Total noise exposure (years) $)^{b)}$ & 0.326 & 0.040 & 0.088 & 0.580 & 0.363 & 0.021 & 0.308 & 0.053 & 0.183 & 0.257 \\
\hline Usage of ear muffs ${ }^{a)}$ & 0.175 & 0.274 & -0.050 & 0.758 & 0.029 & 0.856 & -0.177 & 0.267 & -0.086 & 0.594 \\
\hline Usage of ear plugs ${ }^{a}$ & 0.067 & 0.679 & -0.006 & 0.968 & 0.048 & 0.765 & 0.000 & 1.000 & 0.249 & 0.117 \\
\hline $\begin{array}{l}\text { Absence of earplugs during } \\
\text { explosion }^{a)}\end{array}$ & 0.231 & 0.146 & 0.031 & 0.849 & 0.170 & 0.288 & 0.292 & 0.064 & 0.120 & 0.455 \\
\hline $\begin{array}{l}\text { Psychological problems after } \\
\text { explosion }^{a)}\end{array}$ & 0.345 & 0.027 & 0.100 & 0.533 & 0.218 & 0.171 & 0.132 & 0.411 & 0.371 & 0.017 \\
\hline Sleep disorders after explosion ${ }^{a}$ ) & 0.333 & 0.034 & 0.038 & 0.816 & 0.181 & 0.258 & 0.139 & 0.385 & 0.438 & 0.004 \\
\hline $\begin{array}{l}\text { Acute tinnitus during and just } \\
\text { after explosion }{ }^{a}\end{array}$ & 0.314 & 0.046 & 0.327 & 0.037 & 0.026 & 0.871 & 0.090 & 0.575 & 0.170 & 0.287 \\
\hline Chronic tinnitus still going on ${ }^{a}$ & 0.346 & 0.027 & -0.142 & 0.374 & 0.053 & 0.743 & -0.095 & 0.114 & 0.120 & 0.456 \\
\hline Hearing loss after explosion ${ }^{a}$ & 0.090 & 0.576 & 0.281 & 0.075 & 0.160 & 0.319 & 0.554 & 0.479 & 0.246 & 0.121 \\
\hline Distance from the explosion site ${ }^{b)}$ & -0.041 & 0.801 & 0.080 & 0.618 & -0.074 & 0.647 & 0.183 & 0.251 & 0.008 & 0.959 \\
\hline Brinkmann Index ${ }^{b)}$ & 0.338 & 0.030 & 0.180 & 0.250 & -0.066 & 0.683 & 0.116 & 0.471 & 0.206 & 0.196 \\
\hline
\end{tabular}

a) Spearman's Correlation Rho Efficient Test was used for statistical analysis. $b$ ) Pearson Correlation Test was used for statistical analysis.

\section{DISCUSSION}

The nonspecific effects, derived to the noise exposition can be divided in: psychological effects; psychosomatic effects; psychosocial effects. The psychological effects are anxiety, depression and conflict condiction. ${ }^{4)}$

The noise deafness, in first time, impair the frequencies included between 3000 and $6000 \mathrm{~Hz}$, particularly it concerns the thigh band of $4000 \mathrm{~Hz}$. Those lesions happen after a prolonged exposition to greater than $80-85 \mathrm{dBA}$. The noise is the cause of the arising of psychosocial effects. These can be classified as: interference on the transmission and understanding of the words, perfect healthy respect the efficiency and the learning capacity of the exposed subject, interference on the duration and quality of the sleep. ${ }^{4)}$

The chronic reactions to an occupational permanent noise are mostly a state of fatigue, lowering of the psychotechnic ability and aggressivity. ${ }^{14)}$ Industrial workers may be exposed to more than one 
source of stress and it is not possible to completely attribute the disturbed nocturnal sleep and changes in heart rate to the effects of loud noise alone. ${ }^{15)}$

In the present study, we investigated the effects of explosive blast trauma on sleep qualities and QOLs of the workers exposed blast trauma during their work. The difference between all items of 10point MSQ and SF-36 items were not significant between two groups. Since very long time had passed from the mentioned explosion in 1997, it was concluded that the effects of acute phase decreased over time. Adaptation process may have played a role in these results. Therefore, negative effects on sleep and QOL were thought to be reduced during this period.

For the study group, worker's education levels being increased; and the workers being adjacent to the explosion site; MSQ results get increased and get worse; and QOLs decreased. Daily noise exposure and total noise exposure time; and Brinkmann Index values being increased; ear plugs being not used; and the workers being adjacent to the explosion site, sleep qualities were negatively affected. Combined use of earmuffs and earplugs in association with a safe distance of over $5 \mathrm{~m}$ from the noise source gives adequate protection against AAT. ${ }^{16)}$ In our study, during explosion, none of the workers used ear muffs; and in workers did not use earplugs during explosion, their sleep qualities reduced.

In workers of the study group who had experienced psychological and sleep problems; and hearing loss after explosion; and also having acute tinnitus just after explosion, sleep parameters according to the MSQ got worse and QOLs of the workers reduced. Although chronic tinnitus which is still going on, does not affect QOLs of the workers; it cause increase and worsening of HS item of MSQ. It shows that, workers are used to live with chronic tinnitus and their QOLs is not affected by chronic tinnitus.

Rios and da Silva investigated the effect of chronic workplace exposure to excessive noise on sleep quality. Active men working 40-hr-a-week in the presence of excessive noise without adequate protection for more than eight years presented with noise-induced hearing loss but their quality or quantity of night sleep was unaffected. Sensori-neural deafness may represent an element of adaptation against noise during sleep. ${ }^{17)}$

It was reported that during the positive phase of bomb blast that the tympanic membrane ruptures. Spalling is a likely explanation for the mechanism of rupture of the tympanic membrane especially in large perforations. Tympanic membrane perforations did not protect the inner ear, the sensorineural deafness producing either a high tone or a flat loss. ${ }^{18)}$ Increasing oxygen free radical reaction may be one of the causes of damage in blast trauma induced hearing loss. ${ }^{19)}$

Treatment of AAT should include the correction of impaired microcirculation and tissue oxygenation in the cochlea. ${ }^{2)}$ Treatment should also be focused on reduction of cellular hyperreaction and removal of metabolites that occur during the high functions of the outer hair cells. ${ }^{20)}$ In guinea pigs, the best therapeutic effect on noise induced hearing loss was achieved with corticoid alone or in combination with hyperbaric oxygenation. ${ }^{2)}$

In our study, it was detected that in workers exposed to blast trauma group, the ones exposed to higher daily and total noise during their work, had more affected in terms of poor sleep qualities. Earplugs being not present during explosion and acute tinnitus being occurred due to explosion; and psychological problems and sleep problems were observed after explosion, MSQ items reduced. Chronic tinnitus caused only HS; and it was concluded that as the workers are used to live with chronic tinnitus, their QOLs are not affected.

We may conclude that although probable adaptation process after the explosion may have been developed, explosive acoustic trauma may affect negatively some items of sleep quality and QOLs of the workers. Because of that, all of the precautionary measures should be taken to prevent explosions in the Ammunition Factories. Workers should be educated to use ear muffs and ear plugs. For postexplosion sleep problems and other psychological problems, psychological support should be given to the workers by the health-team and their families.

\section{REFERENCES}

1) Kuokkanen, J., Aarnisalo, A. A. and Ylikoski, J. (2000) Efficiency of hyperbaric oxygen therapy in experimental acute acoustic trauma from firearms. Acta Otolaryngol. Suppl., 543, 132-134.

2) Psillas, G., Pavlidis, P., Karvelis, I., Kekes, G., Vital, V. and Constantinidis, J. (2008) Potential efficacy of early treatment of acute acoustic trauma with steroids and piracetam after gunshot noise. Eur Arch Otorhinolaryngol., 2008 May 8 (Epub ahead of print). 
3) Attanasio, G., Buongiorno, G., Piccoli, F., Mafera, B., Cordier, A., Barbara, M., Filipo, R. (2001) Laser Doppler measurement of cochlear blood flow changes during conditioning noise exposure. Acta Otolaryngol., 121, 465-469.

4) Cosa, M. and Cosa, G. (1989) Annoyance, disturbance and damage caused by noise and vibrations. Ann. Ig., 1, 133-156.

5) Ylikoski, J. (1987) Audiometric configurations in acute acoustic trauma caused by firearms. Scand. Audiol., 16, 115-120.

6) Mrena, R., Savolainen, S., Pirvola, U. and Ylikoski, J. (2004) Characteristics of acute acoustical trauma in the Finnish Defence Forces. Int. J. Audiol., 43, 177-181.

7) Oeken, J. (1998) Distortion Product Otoacoustic Emissions in acute acoustic trauma. Noise Health, 1, 56-66.

8) Gitanjali, B. and Dhamotharan, R. (2003) Effect of occupational noise on the nocturnal sleep architecture of healthy subjects. Indian J. Physiol. Pharmacol., 47(4), 415-422.

9) Murillo, I. C. (2007) How does noise affect us? In our health, life styles and environs. Rev. Enferm., 30, 13-16, 18-20.

10) Alster, J., Shemesh, Z., Ornan, M. and Attias, J. (1993) Sleep disturbance associated with chronic tinnitus. Biol. Psychiatry, 34, 84-90.

11) Ware, J. E. Jr. and Sherbourne, C. D. (1992) The MOS 36-item short-form health survey (SF-36). I. Conceptual framework and item selection. Med. Care., 30, 473-483.

12) Nomura, K., Nakao, M. and Yano, E. (2005) Hearing loss associated with smoking and occupa- tional noise exposure in a Japanese metal working company. Int. Arch. Occup. Environ. Health, 78, 178-184, (Epub 2005 Mar 11).

13) $52^{\text {nd }}$ WMA General Assembly (2000) World Medical Association Declaration of Helsinki: ethical principles for medical research involving human subjects. JAMA, 284, 3043-3049.

14) Fournier, E. (1992) Non-auditory responses to noise. Objective estimation. Experimental and individual analyses Bull. Acad. Natl. Med., 176, 373-385.

15) Gitanjali, B. and Ananth, R. (2003) Effect of acute exposure to loud occupational noise during daytime on the nocturnal sleep architecture, heart rate, and cortisol secretion in healthy volunteers. J. Осcup. Health, 45, 146-152.

16) Savolainen, S. and Lehtomäki, K. M. (1997) Impulse noise and acute acoustic trauma in Finnish conscripts. Number of shots fired and safe distances. Scand. Audiol., 26, 122-126.

17) Rios, A. L. and da Silva, G. A. (2005) Sleep quality in noise exposed Brazilian workers. Noise Health, 7, 1-6.

18) Pahor, A. L. (1981) The ENT problems following the Birmingham bombings. J. Laryngol. Otol., 95, 399-406.

19) Liu, Z. (1992). Experimental study on the mechanism of free radical in blast trauma induced hearing loss. Zhonghua Er Bi Yan Hou Ke Za Zhi, 27, 24-26, 61.

20) Lamm, K. and Arnold, W. (1999) Successful treatment of noise-induced cochlear ischemia, hypoxia, and hearing loss. Ann. N.Y. Acad. Sci., 884, 233 248. 